\title{
Analysis of Split Magnetic Fluid Plane Sealing Performance
}

\author{
Hui-tao Zhang ${ }^{1,2}$ and De-cai $\mathrm{Li}^{1 *}$ \\ ${ }^{1}$ School of Mechanical, Electronic and Control Engineering, Beijing Jiaotong University, Beijing 100044, China \\ ${ }^{2}$ School of Computer Science, Shijiazhuang University, Shijiazhuang 050035, China
}

(Received 18 May 2016, Received in final form 6 October 2016, Accepted 2 November 2016)

\begin{abstract}
Split magnetic fluid sealing is a combination of magnetic fluid rotary and plane sealing. Using the theory of equivalent magnetic circuit design as basis, the author theorized the pressure resistance performance of magnetic fluid plane sealing. To determine the pressure resistance of magnetic fluid plane sealing, the author adopted the method of finite element analysis to calculate the magnetic field intensity in the gap between plane sealing structures. The author also analyzed the effect of different sealing gaps, as well as different ratios between the sealing gap and tooth and solt width, on the sealing performance of split magnetic fluid. Results showed that the wider the sealing gap, the lower the sealing performance. Tooth width strongly affects sealing performance; the sealing performance is best when the ratio between tooth width and sealing gap is 2 , whereas the sealing performance is poor when the ratio is over 8 . The sealing performance is best when the ratio between the solt width and sealing gap is 4 , indicating a slight effect on sealing performance when the ratio between the solt width and sealing gap is higher. Theoretical analysis and simulation results provide reference for the performance evaluation of different sealing equipment and estimation of critical pressure at interface failure.
\end{abstract}

Keywords : split magnetic fluid sealing, structure parameters, sealing performance, finite element

\section{Introduction}

As a functional material, magnetic fluid not only possesses the fluidity of a liquid but also the magnetism of a solid material. Under the effect of a magnetic field, magnetic fluid presents many features not exhibited by a normal liquid [1-5]. Magnetic fluid sealing technology uses the response of high saturation magnetic fluid to magnetic field to seal the related equipment [6-10]. The advantages of magnetic fluid include zero leakage, long life, high reliability, self-restoration feature, small torque variation under high and low temperature, and simple structure; it can also withstand high speed apart from being completely non-polluting and easily manufactured [11-16]. At present, the sealing technology of magnetic fluid has received much attention and developed rapidly. $\mathrm{Li}$ established the principle of magnetic fluid sealing and deduced the pressure resistance formula [17]. Yang and Li designed a magnetic fluid sealing structure with multistage magnetic source to improve pressure resistance per-

(C)The Korean Magnetics Society. All rights reserved.

*Corresponding author: Tel: +8613011039668

Fax:+86010 51684006, e-mail: 12116317@bjtu.edu.cn; dcli@bjtu.edu.cn formance [18]. Li et al. applied the magnetic fluid sealing technology to single crystal furnace equipment and achieved good results [19]. Despite such results, the sealing structure of magnetic fluid is an integrated part of the axle, thus increasing the difficulty of replacement, maintenance, and testing. Particularly, in large equipment, many parts are connected to the shaft, and the sealing device is in the middle position of the shaft.

Split magnetic fluid sealing technology is proposed as a solution to this problem. In 2007, Boyson proposed the reliability issue of applying split-type sealing technology in centrifugal fluid device [20]. When the seal is invalid, only the sealing unit must be dismantled from the whole equipment, saving manpower and other resources. The interface of the upper and lower halves of the cylindrical shell of the split magnetic fluid sealing part is a planar structure, which involves the plane sealing technology of magnetic liquid. This technology is the extension of rotary sealing technology, and its capability to withstand pressure directly affects the sealing performance of the whole equipment. Therefore, the plane sealing of magnetic fluid is one of the key technologies of split magnetic fluid sealing, and studying the effect of different sealing gaps and ratios between the sealing gap and tooth and solt 
width on the sealing performance of split magnetic fluid is critical to the design of the entire sealing equipment.

The author combined the plane sealing technology of magnetic fluid and rotary sealing technology and proposed a split magnetic fluid sealing structure. The author theorized the pressure resistance performance of magnetic fluid sealing using the theory of equivalent magnetic circuit as basis. The author adopted the finite element method analysis and conducted simulation to calculate the magnetic field intensity in the gap of plane sealing structures to determine the pressure resistance performance of the magnetic fluid. The author also analyzed the effect of different sealing gaps and ratios between the sealing gap and tooth and solt width on the sealing performance of split magnetic fluid.

\section{Theoretical Analysis of the Magnetic Fluid Sealing Performance}

\subsection{Structure of magnetic fluid plane sealing and equivalent magnetic circuit}

Figure 1 illustrates the section view of a split magnetic fluid sealing structure at the place of the pole shoe. The figure shows that the split magnetic fluid sealing is the combination of magnetic fluid rotary and plane sealing. The enlarged view presents a three-toothed magnetic fluid plane sealing structure, and its equivalent magnetic circuit is shown in Fig. 2, in which the material permanent magnet is Nd-Fe-B permanent magnet, the magnetic conductivity is $\mu_{\mathrm{m}}$, the magneto motive force is $F_{c}$, the residual magnetism is $B_{r}$, the coercivity is $H_{c}$, the thickness of the magnet is $l_{m}$, the reluctance is $R_{m}$, and the formula is $R_{m}=l_{m} / \mu_{m} S_{m}=l_{m} H_{c} / B_{r} S_{m}$. The reluctance of the seal gaps filled with magnet fluid is indicated by $R g 1$, $R g 2, R g 3, R g 1 ', R g 2 ', R g 3$ ', respectively. The magnetic

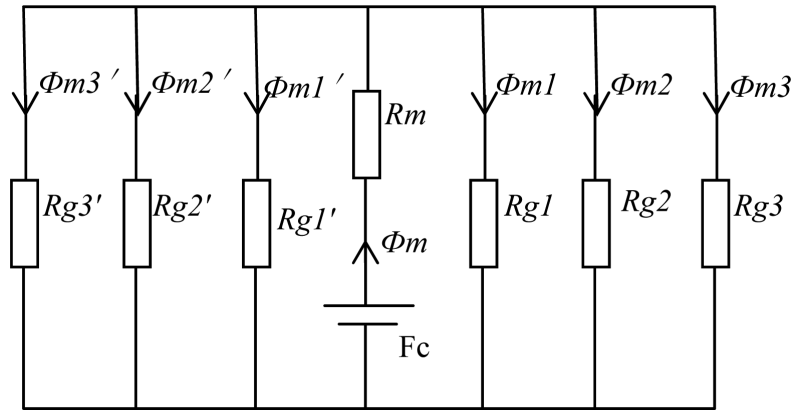

Fig. 2. Equivalent magnetic circuit.

flux in the seal gaps is indicated by $\Phi m 1, \Phi m 2, \Phi m 3$, $\Phi m 1^{\prime}, \Phi m 2^{\prime}, \Phi m 3^{\prime}$. As shown in Fig. 1, the magnetic fluid plane sealing is the unfolded view of the magnetic fluid rotary sealing, and the magnetic circuit is composed of permanent magnet, pole shoe, magnetic fluid, and half shell. The half shell is equivalent to the unfolded view of the rotation axis. As the source of the entire magnetic circuit, the permanent magnet supplies magnetic flux, which traps the magnetic fluid in the gap between the pole shoe and the half shell. Under the effect of magnetic force, the magnetic fluid can withstand the differential pressure on both sides to achieve the sealing [21].

\subsection{Theoretical calculation of magnetic fluid plane sealing performance}

The formula to approximately calculate the pressure resistance of magnetic fluid static sealing $[22,23]$ is

$$
\Delta p=\mu_{0} M_{s} \Sigma_{i=1}^{N}\left(H_{\max }^{i}-H_{\min }^{i}\right)=M_{s} \Sigma_{i=1}^{N}\left(B_{\max }^{i}-B_{\min }^{i}\right),
$$

where $\mu_{0}$ and $M_{s}$ are the vacuum permeability and saturation magnetization of magnetic fluid, respectively, $H_{\max }^{i}$ and $H_{\min }^{i}$ are respectively the maximum and minimum
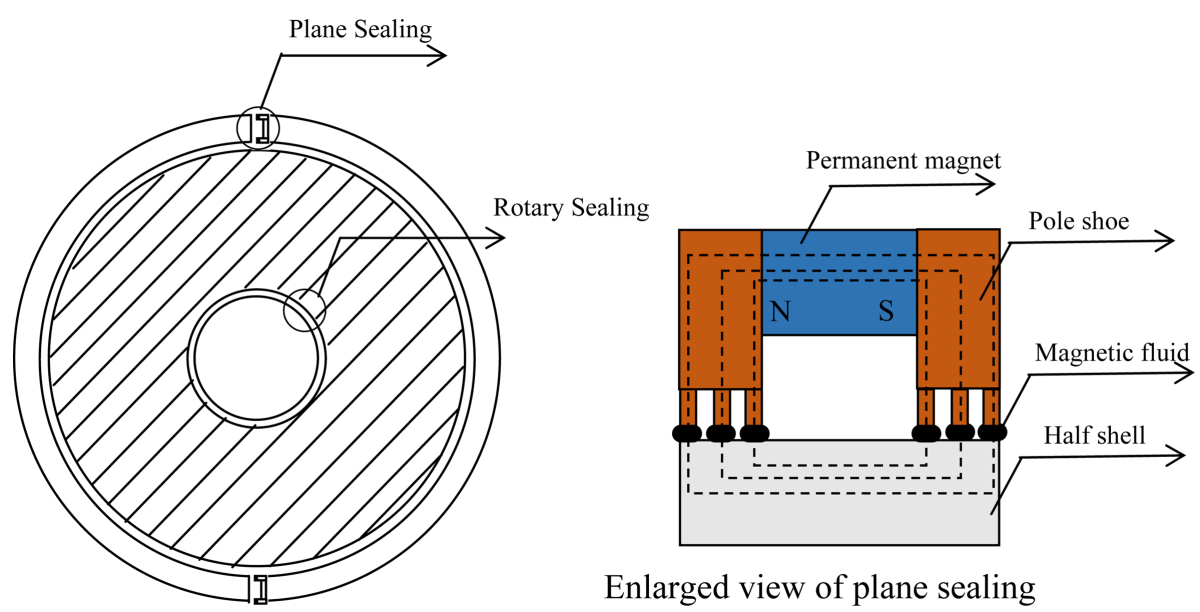

Enlarged view of plane sealing

Fig. 1. (Color online) Section view of split magnetic fluid sealing structure at the place of pole shoe. 
value of the magnetic field strength at the sealing gap between the pole shoe and half shell at the stage $\mathrm{i}$ of the magnetic fluid plane sealing structure, $B_{\max }^{i}$ and $B_{\min }^{i}$ are respectively the maximum and minimum value of the magnetic induction of the magnetic field intensity at the sealing gap between the pole shoe and half shell at the stage $i$ of the magnetic fluid plane sealing structure, and $\mathrm{N}$ is the total stages of the sealing. Drawing on Formula (1), the differential pressure resistance of the magnetic fluid plane sealing can be calculated if the total magnetic flux density difference is definite.

The formula of Kirchhoff's first law is given as follows:

$$
\Sigma_{i} \varnothing_{i}=0,
$$

where the sum of the magnetic flux of each magnetic circuit is zero.

Kirchhoff's second law is expressed as

$$
\Sigma_{i} U_{m i}=\Sigma_{k} F_{m k},
$$

where $U_{m i}$ is the magnetic pressure drop of the magnetic circuit; $F_{m k}$ is the magneto motive force of the magnetic circuit; $U_{m i}=H_{i} l_{i}$, whose position direction is the same as the direction of the magnetic field; $F_{m k}=l_{k} N_{k}$, whose position direction is the same as the direction of the current.

Magnetic Ohm's Law is

$$
\varnothing=\frac{N I}{\frac{1}{\mu S}} .
$$

$R=\frac{1}{\mu S}$ is the reluctance in the magnetic circuit.

Figure 3 is the magnetic curve model of Fig. 1. The model is established for the magnetic curve passing through each pole tooth; the magnetic curves at both sides of pole tooth are simplified as a curve, and the magneto

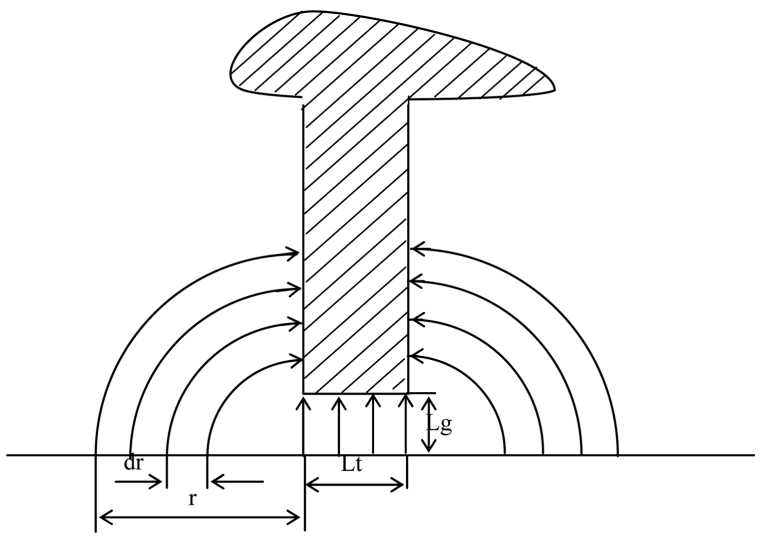

Fig. 3. Magnetic curve model. resistance in the middle part of the vertical is assumed $R$ $=1 / \mu S$.

Assuming that the magneto resistance of the magnetic curve at both sides is $R_{2}$, and $L$ is the length of the pole shoe, then

$$
d R_{2}=\frac{\pi r}{\mu_{0} d_{r} L} .
$$

Assuming that $t=\int \frac{1}{d R_{2}}$,

$t=\int \frac{1}{d R_{2}}=\frac{\mu_{0} L}{\pi} \int \frac{1}{r} d_{r}$,

$R g_{1}=\frac{1}{\frac{1}{R_{1}}+t}$.

Therefore, $R g_{1}=R g_{2}=R g_{3}=R g_{1}{ }^{\prime}=R g_{2}{ }^{\prime}=R g_{3}{ }^{\prime}$,

$R_{g}=\frac{R g_{1}}{6}$.

The magnetic flux in the magnetic circuit is

$\varnothing_{m}=\frac{F_{c}}{R_{m}+R_{g}}$.

The calculation is based on the assumption that the magnetic flux $\varnothing_{p t}$ at each pole tooth is the same.

$$
\varnothing_{p t}=\frac{\varnothing_{m}}{6}
$$

Therefore, the magnetic induction at each pole tooth is $B_{p t}$,

$$
B_{p t}=\frac{\varnothing_{p t}}{s} .
$$

The theoretical pressure resistance value of the split magnetic fluid seal equipment can be obtained when Formula (11) is substituted into Formula (1).

\section{Establishment of Finite Element Model}

The distance should be minimized between the upper and lower halves of the shell, i.e., the height of the pole shoe, the height of pole tooth, and the sealing gap because the axis needs to keep the axle neutral to the split magnetic fluid sealing equipment. In addition, the magnet possesses magnetic range. The finite element model is established (see Fig. 4) by setting the area as a symmetric semicircular with a symmetrical structure of the upper and lower parts. The magnetic fluid is used as an oilbased magnetic liquid with saturated magnetization of $400 \mathrm{G}$. The displacement of all the nodes of the model is 


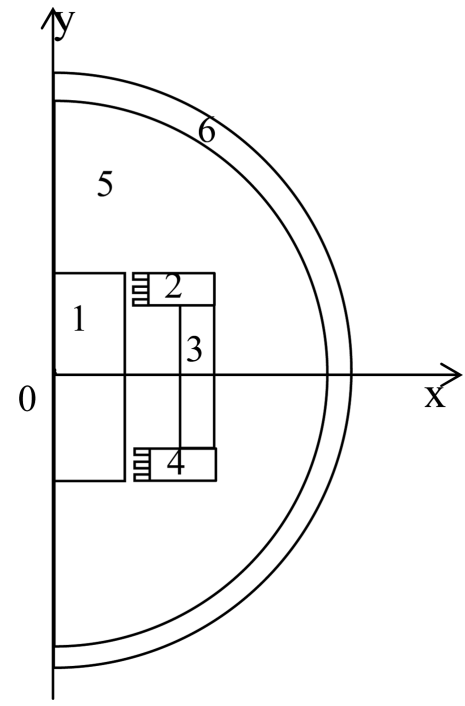

1. Half shell 2. Pole shoe 3. Permanent magnet 4. Pole shoe 5. Air field 6. Infinity field

Fig. 4. Schematic model of finite element model.

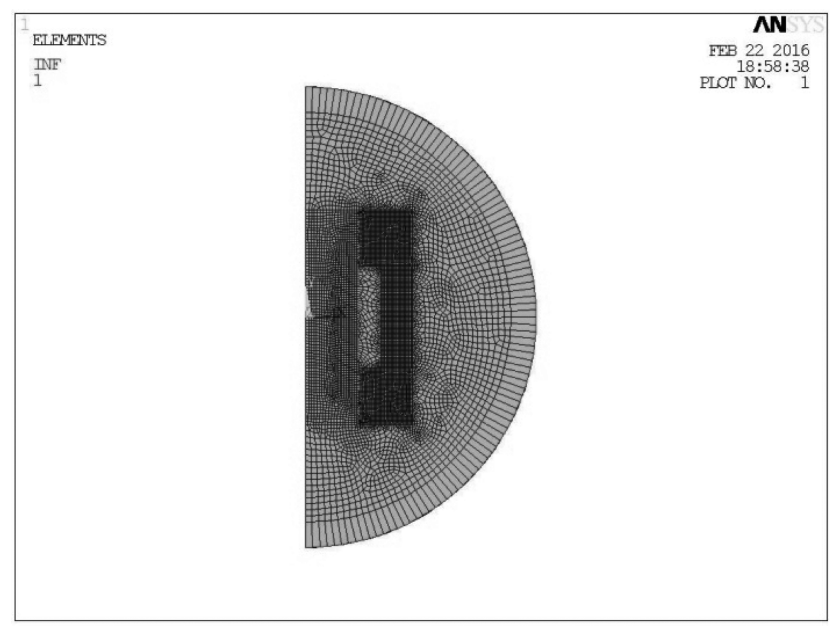

Fig. 5. Mesh division of finite element model.

constrained and fixed. The mesh near the pole tooth is refined during the calculation of the magnetic induction strength to obtain high accuracy. The type of the unit adopted is PLANE53, containing a total of 24,242 nodes (see Fig. 5). The outer semi annular region of the boundary condition is an infinite element.

The magnetic induction strength of split magnetic fluid sealing is simulated for calculation under the circumstance that the parameters of gap, tooth width, and solt width are variable, ensuring that the effect on the pressure resistance of split magnetic fluid sealing can be obtained. In this work, the pressure resistance is calculated for cases in which the gap is $0.1,0.2,0.4$, and $0.8 \mathrm{~mm}$. The ratio of tooth width and gap is $1,2,4$, and 8 , whereas, that of solt
Table 1. Table of parameters to calculate sealing structure.

\begin{tabular}{lccc}
\hline \hline \multicolumn{1}{c}{ Parameter } & Symbol & $\begin{array}{c}\text { Value of } \\
\text { parameter }\end{array}$ & Unit \\
\hline Coercivity of permanent magnet & $H_{c}$ & 930000 & $\mathrm{~A} / \mathrm{m}$ \\
Remanence of permanent magnet & $B_{r}$ & 1.28 & $\mathrm{~T}$ \\
Length of permanent magnet & $L_{m}$ & 0.01 & $\mathrm{~m}$ \\
Length of permanent magnet section & $L_{a}$ & 0.04 & $\mathrm{~m}$ \\
Height of permanent magnet section & $L_{b}$ & 0.003 & $\mathrm{~m}$ \\
Initial tooth width of pole shoe & $L_{t}$ & 0.0004 & $\mathrm{~m}$ \\
Initial solt width of pole shoe & $L_{s}$ & 0.0008 & $\mathrm{~m}$ \\
Length of pole shoe & $L$ & 0.04 & $\mathrm{~m}$ \\
Initial gap & $L g$ & 0.0002 & $\mathrm{~m}$ \\
Saturated magnetization of magnetic fluid & $M$ & 400 & $\mathrm{G}$ \\
Vacuum permeability & $\mu_{0}$ & $4 \Pi \times 10^{-7}$ & $\mathrm{~T} \cdot \mathrm{m} / \mathrm{A}$ \\
\hline
\end{tabular}

width and gap is $2,4,8$, and 16 . Thus, the effect of the parameters of gap, tooth width, and solt width on the performance of split magnetic fluid plane sealing can be obtained. Table 1 presents the theoretical calculation and simulated parameters of split magnetic fluid plane sealing.

\section{Result and Analysis}

\section{1, Effect of sealing gap on sealing performance}

Figure 6 indicates that along with the increase of the sealing gap, the magnetization curve is changed from a regular peak-trough curve into an irregular graph; consequently, the difference between the peak value and trough value decreases. According to the pressure resistance formula of split magnet fluid plane sealing performance, the wider the sealing gap, the poorer the sealing performance of pressure resistance.

The theoretical differential pressure in Fig. 7 is obtained by substituting the parameters of plane sealing structure in Table 1 into Formula (1) to Formula (11). For the theoretical calculation, the upper limit of the integral is obtained as $1 / 3$ of the solt width plus $1 / 2$ of the tooth width, and the lower limit is taken as $1 / 2$ of the tooth width. As shown in the theoretical differential pressure curve, the differential pressure decreases along with the increasing sealing gap. This outcome is because the increase of gap increases the reluctance of the magnetic circuit, which, in turn, decreases the total flux, thereby reducing the sealing pressure resistance performance of the equipment. The total magnetic intensity difference is obtained through Ansys simulation of the parameters of the plane sealing structure in Table 1. The simulated differential pressure curve is obtained by substituting the total magnetic intensity difference into Formula (1). As shown in the simulated differential pressure curve, along 

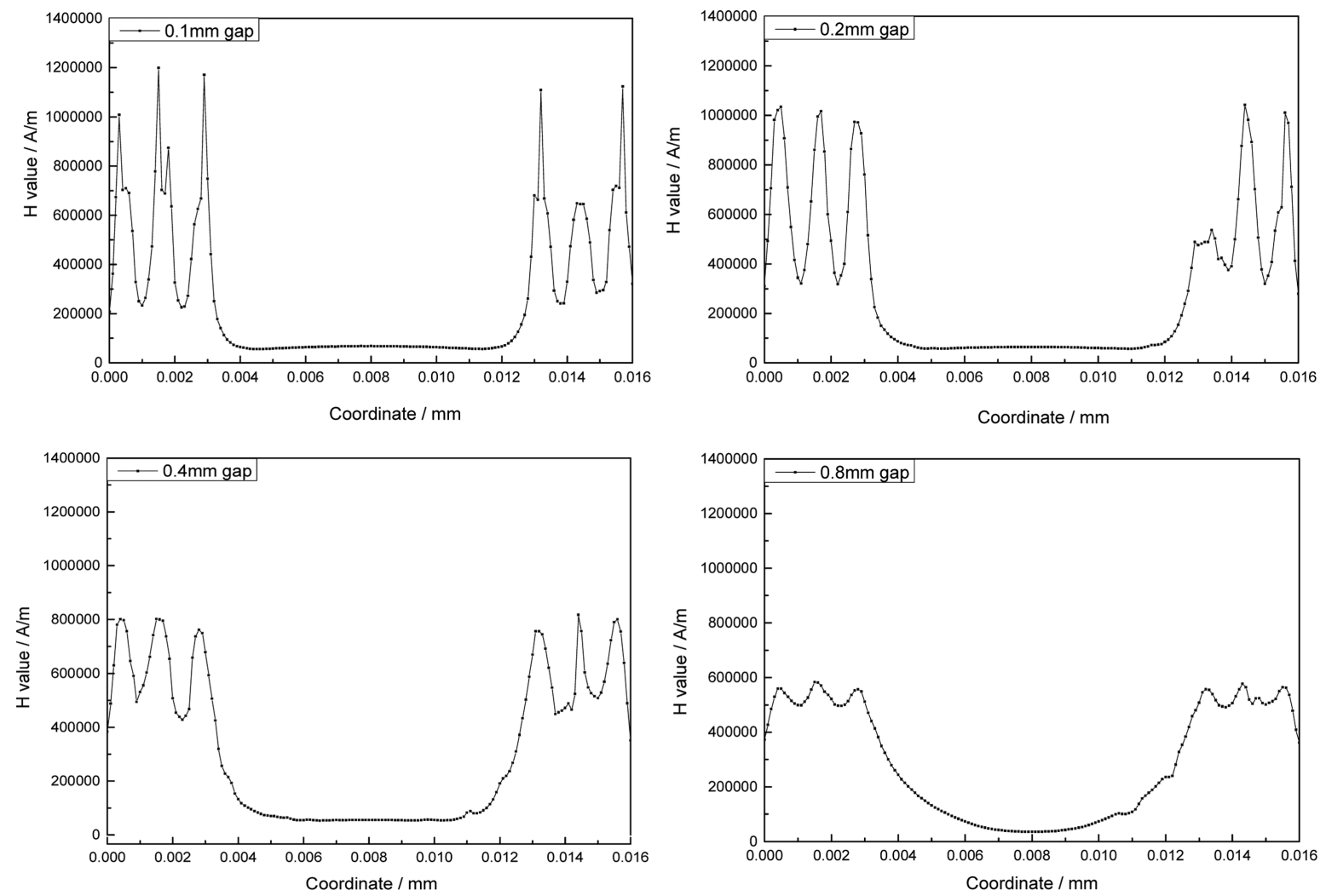

Fig. 6. Analog distribution of value $H$ when the sealing gap is $0.1,0.2,0.4$, and $0.8 \mathrm{~mm}$.

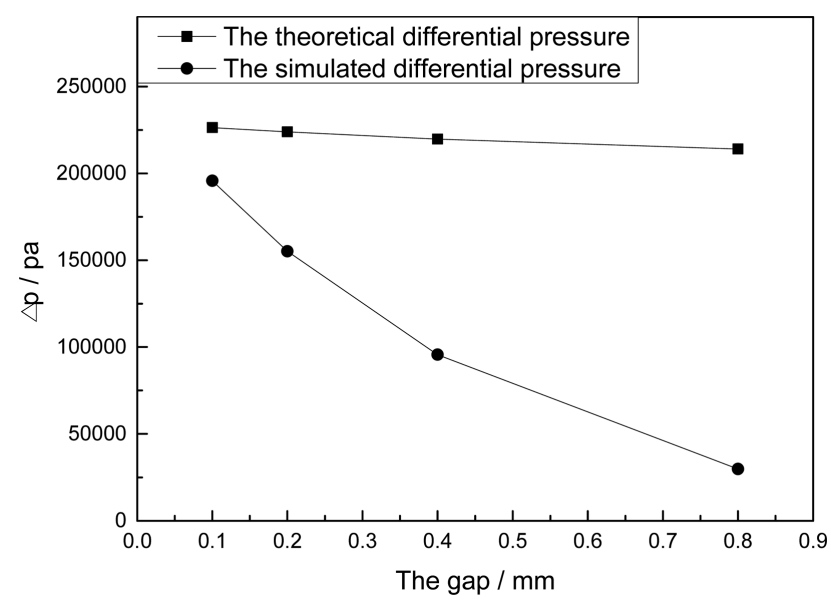

Fig. 7. Comparison chart of theoretical differential pressure and simulated differential pressure for different sealing gaps.

with the increase of sealing gap, the differential pressure gradually decreases and the decline becomes smaller. The overall differential value is also less than the theoretical differential pressure, which is caused by the magnet leakage at the connection of the permanent magnet and the pole shoe. The magnet leakage of the permanent magnet is ignored during theoretical calculation.

\subsection{Effect of the ratio of pole shoe tooth width and gap on sealing performance}

As shown in Fig. 8, when the ratio of the pole shoe tooth width and the gap is 1 or 2 , the variation of magnetic induction intensity curve is regular. When the ratio of the pole shoe tooth width and the gap is 2, the distance between the peak and trough becomes longer, indicating that the sealing pressure resistance performance is enhanced. When the ratio of the pole shoe tooth width and the gap is 4 , the magnetic induction intensity curve becomes more irregular. Such irregularity becomes more apparent when the ration is 8 , indicating that when the pole shoe width reaches a certain degree, the sealing pressure resistance performance begins to decrease after peaking. The decrease of the magnetic flux density in the sealing gap, that is, the decrease of magnetic field intensity, reduces sealing performance.

The theoretic differential pressure curve in Fig. 9 is obtained by substituting the parameters of the plane sealing structure in Table 1 into Formula (1) to Formula (10) when changing the parameter of tooth width, although the sealing gap and solt width is maintained as initial value. As shown in the theoretic differential pressure curve, the 

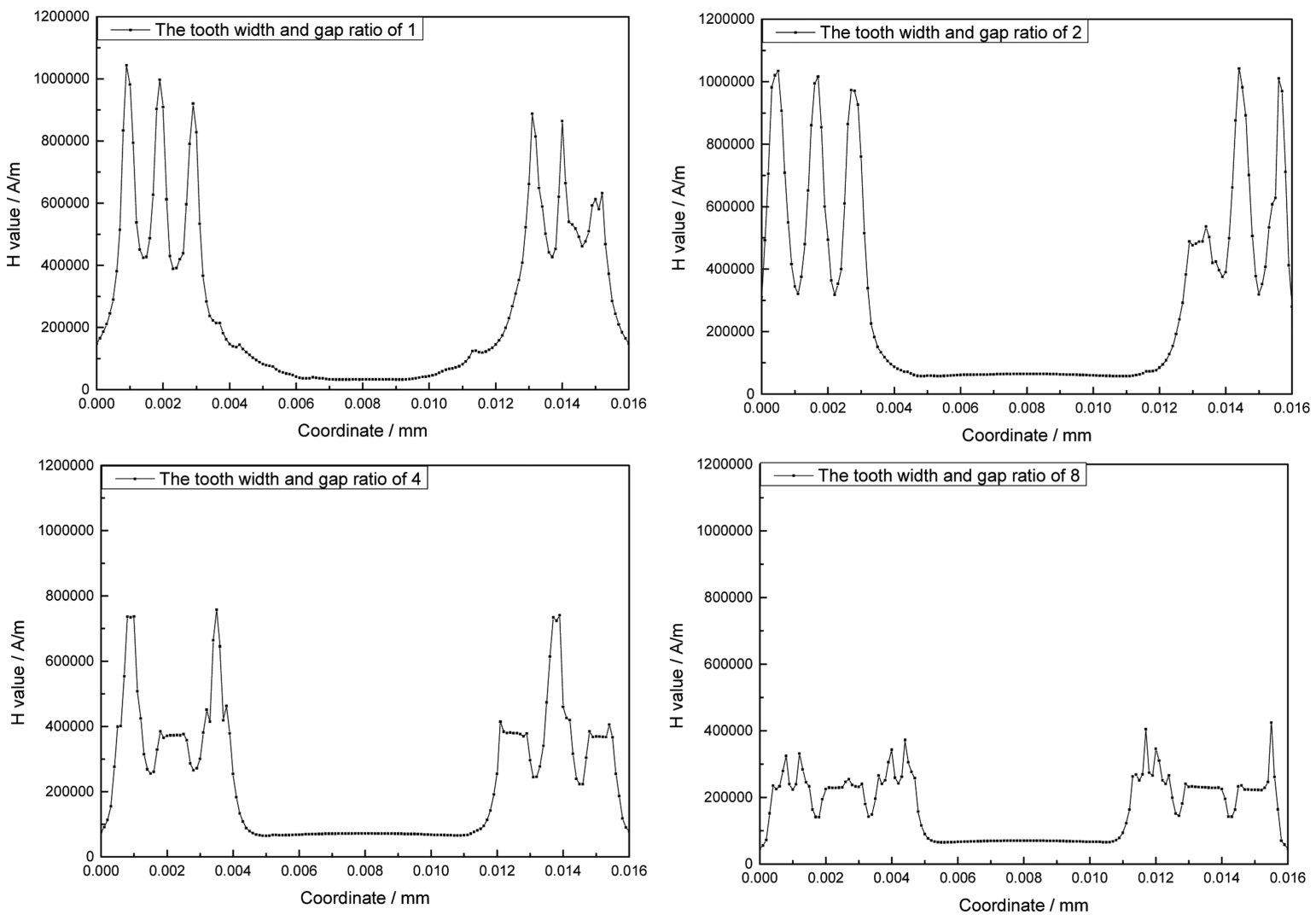

Fig. 8. Analog distribution of value $H$ when the ratio of tooth width and gap is $1,2,4,8$.

tooth width greatly affects the split magnetic fluid plane sealing performance. Along with the increase of the ratio of tooth width and gap, the sealing pressure resistance performance decreases rapidly, because the increase of the tooth width increases the magnetic field area going through the sealing gap. Thus, the rapid decrease of the total magnetic flux in the sealing gap is the result of the rapid decrease of total magnetic flux in the sealing gap. The simulated differential pressure curve is obtained by substituting the total magnetic field density difference value into Formula (1), in which the total magnetic field density difference value is obtained through ANSYS simulation of the parameters of plane sealing structure in Table 1 when changing the parameter of tooth width while maintaining the sealing gap and solt width as initial value. The simulated differential pressure curve shows that along with the increase of the tooth width, the differential pressure initially exhibits a slight increase then shows a tendency of slow decrease. When the ratio of the tooth width and the gap is 8 , the value of the simulated differential pressure is close to the result of theoretical calculation, indicating that when the ratio of the tooth width and the gap of the split magnetic fluid plane sealing

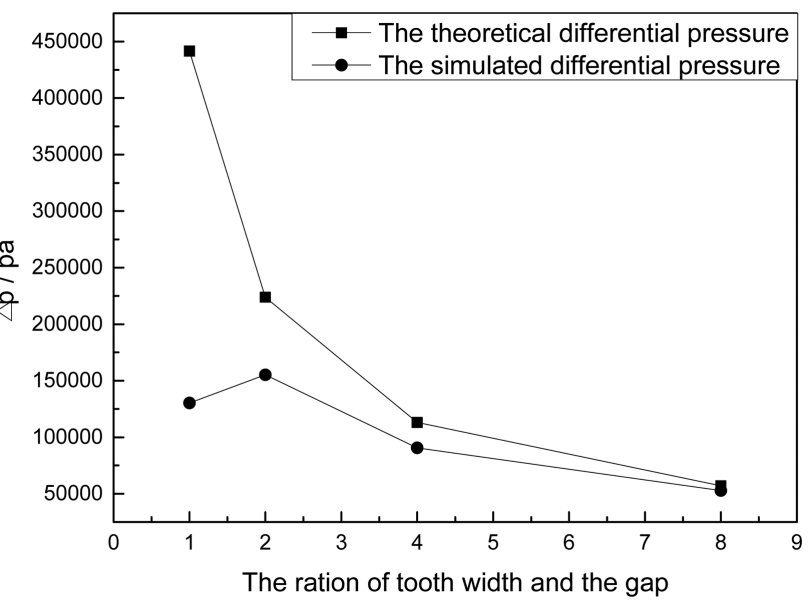

Fig. 9. Comparison chart of theoretical and simulated differential pressure for different ratios of tooth width and sealing gap.

structure is higher than 8 , the magnetic flux applied to the permanent magnet is low, the magnetic flux of the magnetic circuit is small, the magnetic field in the sealing gap is weak. All these outcomes lead to poor sealing performance. 

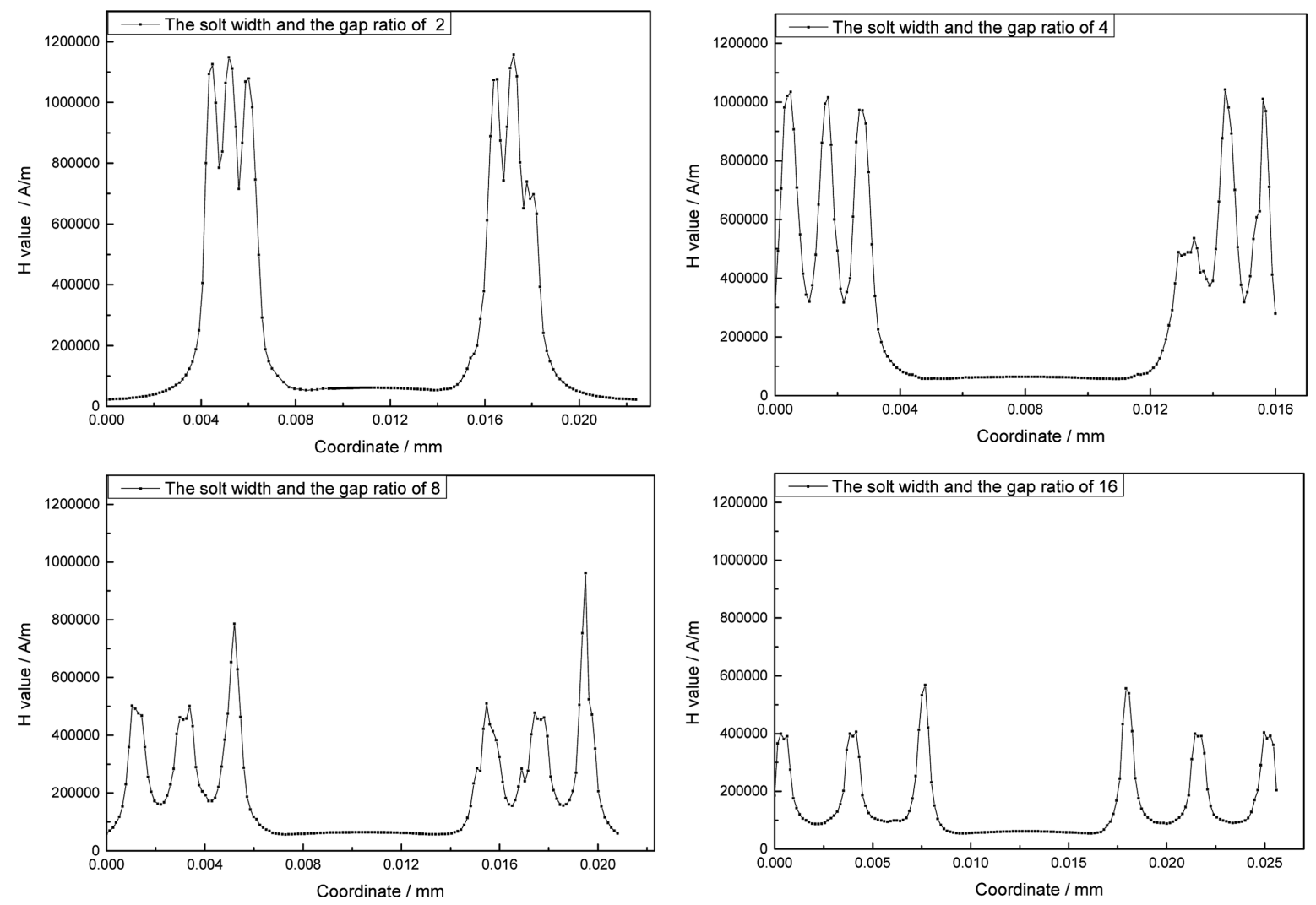

Fig. 10. Analog distribution of value $H$ when the ratio of solt width and gap is $2,4,8,16$.

\subsection{Effect of the ratio of solt width and gap on sealing performance}

As shown in Figs. 10 and 11, for the split magnetic fluid plane sealing structure, the sealing performance exhibits a slight increase at the beginning of the increase of the pole shoe width because the multi-tooth sealing

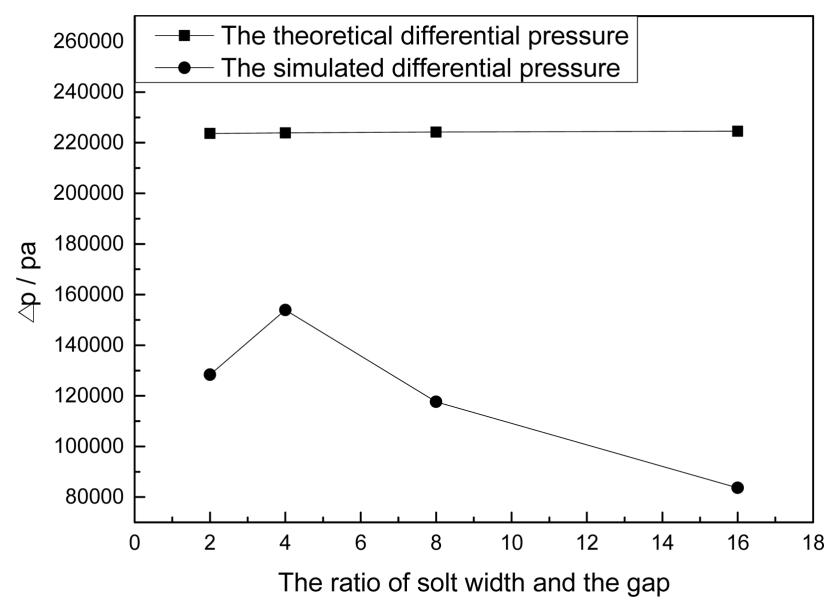

Fig. 11. Comparison chart of theoretical and simulated differential pressure for different ratios of solt width and sealing gap. structure becomes a single-tooth structure when the solt width limit is assumed zero, resulting in poor sealing performance. However, along with the increase of the solt width, the magnetic field is formed in the tooth groove, and the gradient increase of the magnetic flux improves the sealing performance. Nevertheless, when the pole shoe solt width reaches a certain degree, the magnetic field in the tooth groove becomes excessively small that it becomes zero. Thus, the change of the sealing pressure resistance performance becomes obscure.

\section{Conclusions}

This paper presents a split sealing structure combining magnetic fluid rotary sealing and plane sealing. The differential pressure formula of split magnetic fluid plane sealing is deduced. The magnet leakage of the permanent magnet causes great difference between the theoretical and simulated differential pressure. Preventing magnet leakage is crucial to improve the sealing performance. The optimal parameters are established by simulation. Theoretical analysis and simulation results provide reference for the performance evaluation of different sealing equipment and the estimation of critical pressure at 
interface failure. This research can solve the problem of large equipment sealing device for easy disassembly and maintenance.

\section{Acknowledgments}

This research was supported by the National Natural Science Foundation of China (Grant No. 51375039), Beijing Natural Science Foundation (Grant No. 4142046) and Program for Changjiang Scholars and Innovative Research Team (Grant No. IRT13046).

\section{References}

[1] J. Zou, X. Li, Y. Lu, and J. Hu, J. Magn. Magn. Mater. 252, 321 (2002).

[2] R. Rosensweig and E. Ferrohydrodynamics, Dover Publications INC, New York (2002).

[3] I. Nakatani, T. Furubayashi, T. Takahashi, and H. Hanaoka, IEEE Trans. J. Magn. in Japan 65, 261 (1987).

[4] Z. Li, J. Magn. Magn. Mater. 252, 327 (2002).

[5] N. C. Popa, I. D. Sabata, I. Anton, I. Potencz, and L. Vékás, J. Magn. Magn. Mater. 201, 385 (1999).

[6] W. L. Lian, L. Qiang, and Y. M. Xuan, Science in China 51, 1203 (2008).

[7] W. Ochoński, Wear 130, 261 (1989).

[8] Y. Mitamura, S. Takahashi, S. Amari, E. Okamoto, S. Murabayashi, and I. Nishimura, Physics Procedia 9, 229
(2010).

[9] L. Matuszewski and Z. Szydlo, Polish Maritime Research 15, 49 (2008).

[10] K. Raj, B. Moskowitz, and R. Casciari, J. Magn. Magn. Mater. 149, 174 (1995).

[11] G. Morton and W. G. Früh, J. Magn. Magn. Mater. 252, 324 (2002).

[12] M. Sun, Large Scale Nitrogenous Fertilizer Industry (2006).

[13] K. Willenborg, S. Kim, and S. Wittig, J. Turbomachinery 123, 815 (2001).

[14] I. Etsion, Y. Kligerman, and G. Halperin, Tribology Transactions 42, 511 (2008).

[15] W. Shapiro, C. Lee, H. Jones, J. Tribology 110, 525 (1987).

[16] Q. Yang, C. F. Gao, and W. Chen, Tribology Transactions 29, 151 (1986).

[17] D. Li, H. Xu, X. He, and H. Lan, J. Magn. Magn. Mater. 289, 419 (2005).

[18] X. L. Yang, Z. L. Zhang, and L. I. Decai, Science China Technological Sciences 56, 2865 (2013).

[19] De-cai, Li, Beijing: Science Press 484 (2003). (in Chinese).

[20] S. Boyson, Sealing Technology 2007, 7 (2007).

[21] T. Liu, Y. Cheng, and Z. Yang, J. Magn. Magn. Mater. 289, 411 (2005).

[22] V. K. Polevikov, Fluid Dynamics 32, 457 (1997).

[23] M. Zhao, J. B. Zou, J. Hu, J. Magn. Magn. Mater. 303, 428 (2006). 